\title{
Impacts of Telemanipulation in Robotic Assisted Surgery
}

\author{
Ignacio Avellino \\ Sorbonne Université, CNRS, ISIR \\ Paris, France \\ avellino@upmc.isir.fr \\ Jérémie Belghiti \\ Hôpital Pitié Salpêtrière \\ Paris, France \\ jeremie.belghiti@aphp.fr
}

\author{
Gilles Bailly \\ Sorbonne Université, CNRS, ISIR \\ Paris, France \\ bailly@upmc.isir.fr
}

\author{
Guillaume Morel \\ Sorbonne Université, CNRS, ISIR \\ Paris, France \\ morel@upmc.isir.fr
}

\author{
Geoffroy Canlorbe \\ Hôpital Pitié Salpêtrière \\ Sorbonne Université \\ Paris, France \\ geoffroy.canlorbe@aphp.fr
}

\author{
Marie-Aude Vitrani \\ Sorbonne Université, CNRS, ISIR \\ Paris, France \\ vitrani@upmc.isir.fr
}

\begin{abstract}
Robotic-assisted Minimally Invasive Surgery (MIS) is adopted more and more as it overcomes the shortcomings of classic MIS for surgeons while keeping the benefits of small incisions for patients. However, introducing new technology oftentimes affects the work of skilled practitioners. Our goals are to investigate the impacts of telemanipulated surgical robots on the work practices of surgical teams and to understand their cause. We conducted a field study observing 21 surgeries, conducting 12 interviews and performing 3 data validation sessions with surgeons. Using Thematic Analysis, we find that physically separating surgeons from their teams makes them more autonomous, shifts their use of perceptual senses, and turns the surgeon's assistant into the robot's assistant. We open design opportunities for the HCI field by questioning the telemanipulated approach and discussing alternatives that keep surgeons on the surgical field.
\end{abstract}

\section{CCS CONCEPTS}

\section{- Human-centered computing $\rightarrow$ Empirical studies in HCI.}

\section{KEYWORDS}

Robotic Assisted Surgery, Minimally Invasive Surgery, da Vinci

CHI 2019, May 4-9, 2019, Glasgow, Scotland UK

(c) 2019 Association for Computing Machinery.

This is the author's version of the work. It is posted here for your personal use. Not for redistribution. The definitive Version of Record was published in CHI Conference on Human Factors in Computing Systems Proceedings (CHI 2019), May 4-9, 2019, Glasgow, Scotland UK, https://doi.org/10.1145/3290605.3300813.
ACM Reference Format:

Ignacio Avellino, Gilles Bailly, Geoffroy Canlorbe, Jérémie Belghiti, Guillaume Morel, and Marie-Aude Vitrani. 2019. Impacts of Telemanipulation in Robotic Assisted Surgery. In CHI Conference on Human Factors in Computing Systems Proceedings (CHI 2019), May 49, 2019, Glasgow, Scotland UK. ACM, New York, NY, USA, 15 pages. https://doi.org/10.1145/3290605.3300813

\section{INTRODUCTION}

"[The design of new technology] alters what is already going on-the everyday practices and concerns of a community of people-and leads to a resettling into new practices, which in turn create new future design possibilities." - Flores et al. [20].

It is well-established that new technology design often leads to desirable and undesirable unanticipated effects [34, 73], both because of the complexity of humans factors (e.g. cognitive and physical abilities, reasoning, affect) and environmental factors (e.g. institutions, equipment, practices). Indeed, people both adapt and adopt technology in ways unexpected to the designer [39]. Studies have shown the complex relationship between the technology and its use, for instance in flight management systems [12] and medical devices $[2,11,55]$. Their findings help rethink and improve technology design [20,39].

We investigate the effects of introducing telemanipulated surgical robots on the existing practices of medical teams. Robotic-assisted Minimally Invasive Surgery (MIS) is an increasingly adopted high-end technology, in 2014 there were more than 652.000 surgical interventions performed with the da Vinci robot [68]. It keeps the clinical benefits of small incisions for patients while overcoming the shortcomings of classic MIS (without robotic assistance) for surgeons. While this technology promised to increase the benefits for patients, a recent analysis [66] and medical studies [26, 57] put this into question. Have we not yet seen the anticipated clinical benefits of robotic surgery because of the changes the technology imposes on work practices? 

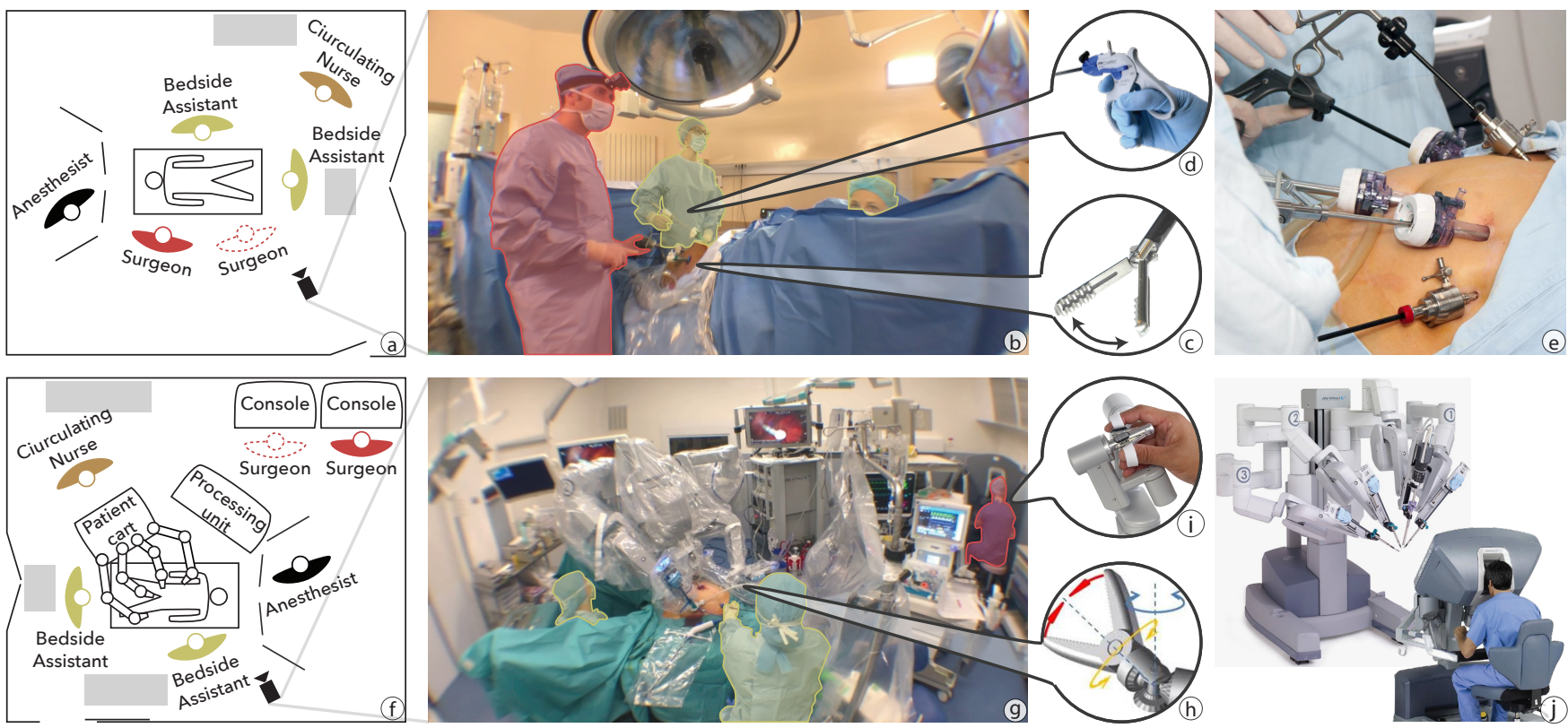

Figure 1: Classic MIS (top) vs. robotic-assisted MIS (bottom) in gynecology. Classic MIS operating room diagram (a) and image from our study (b) where a surgeon and two assistants work face-to-face. Mechanical tools have low dexterity, with only two DoF, grasp and rotate (c), activated by pulling a trigger and a rotation knob (d). Surgeons insert instrument through small holes (e). Robotic-assisted MIS operating room diagram (f) and image from our study (g) where a surgeon works at the console and two assistants at the bed side. Free-hand moving joysticks (i) control highly dexterous tools with six DoF (h) on the robotic $\operatorname{arms}(\mathrm{j})$.

We observed 21 surgical interventions, conducted 12 interviews with medical staff members, and performed 3 data validation sessions with surgeons. We focused on the differences between classic and robot-assisted MIS to understand the complexity of interacting with the new technology and ultimately shed light on the design factors that lead to the unanticipated effects of the technology. We performed a thematic analysis of our data to gain insights on the reasons behind the changes that the technology brings [7].

We find an overarching theme which captures to a large extent the essence of the impacts on existing work practices: the physical separation between surgeons, their teams, and patients impacts the existing practices of surgical teams. This distance increases the control and autonomy that surgeons have on surgical gestures although it secludes them, decreasing situational awareness as well as other people's perception of their presence. Surgeons mitigate the loss of haptic feeling and the restricted visual access to their teams by shifting their use of perceptual senses, relying more on verbal exchanges. The bedside assistant role is redefined, providing less assistance to the surgeon and more to the robot. Skill acquisition is also affected as medical interns learn surgical skills by watching instead of doing. Based on these findings, we discuss a new design dimension for surgical robots: the distance from the surgeons to the surgical field.
We believe that minimizing this distance can retain the core benefits of robotics without hindering the subtle relationship between the different members of the medical team, ultimately making surgery minimally invasive not only for patients but also for medical teams.

\section{BACKGROUND}

We describe what surgery is and introduce both classic and robotic-assisted MIS to provide background for our study.

Surgery lets doctors investigate and treat pathological conditions on patients with the goal of improving their health. Besides the anesthetist, there are four main roles during a surgery (Figure 1-a,f):

- operating surgeon: operates on the patient;

- bedside assistant: assists the main operator;

- scrub instrumentalist: handles tools in the sterile field;

- circulating instrumentalist: handles tools outside this field.

The operating surgeon is usually a surgeon and the bedside assistant a medical intern, though both may switch roles. The scrub instrumentalist is usually a nurse or a medical intern, the circulating instrumentalist is usually a nurse. 
Senior surgeons let medical interns operate under their supervision to teach them through what is known as Bedside Teaching [50]. This is a multi-sensory experience for interns: they observe surgeon's actions and facial expressions and gaze direction; they hear surgeons verbalize their strategy and the rationale for their actions; and surgeons can grab their hands to teach them hands-on surgical gestures.

Open surgery is the earliest type of intervention. Surgeons perform a large incision and access the operating site with their hands, having an unrestricted view and feeling of touch. A large incision however has important consequences for patients, including a long recovery time, high risk of infection and visible scaring. To eliminate the need for large incisions, minimally invasive procedures were developed.

Classic Minimally-Invasive Surgery (MIS) became widely adopted after the first laparoscopic cholecystectomy in 1987 [15]. In classic MIS surgeons open small holes and insert trocars, tubular devices that allow tools to be inserted and removed while restricting air flow. Surgeons use elongated mechanical instruments to operate and an endoscope (camera) to see inside the body through a 2D monitor. MIS has become the routine procedure for interventions such as cholecystectomy and appendectomy [52, 70].

Small incisions bring a number of benefits for patients including reduced pain, hospital stay, discomfort, and a quicker return to work $[1,22,58]$, but they impact the surgeons' work. First, surgeons suffer from the fulcrum effect [53]: as tools pivot about the incision point (Figure 1-e), their movement is mirrored in a counter-intuitive way [69] and forces are amplified between the distal and proximal ends. Furthermore, movement is limited from six to four degrees (three of rotation but only one of translation), and dexterity is hindered from using rigid mechanical instruments (Figure 1-d,c).

Second, surgeons suffer from poor ergonomics. Instruments demand excessive wrist flexion and a high ratio of handle-to-tip force transmission causing fatigue, discomfort, and even hand paresthesias [3]. The standing posture maintained for a long time can lead to health issues ranging from neck and hand pain [4] to serious conditions such as carpal tunnel syndrome, disc problems, tennis elbow, or shoulder muscle spasm [56].

Third, surgeons suffer from hindered perception and motor coordination. Haptics are reduced as surgeons do not touch tissues directly, althou they can still determine object features like shape, texture and consistency [5]. Visual-motor coordination is affected from seeing through using a 2D monitor [27].

Robotic-Assisted MIS relies on a surgical robot, " $a$ powered, computer-controlled manipulator with artificial sensing that can be programmed to move and position tools to carry out a wide range of surgical tasks (telemanipulator)" [16]. Surgical robots come into the operating room to overcome the shortcomings of classic MIS, but also with the promise of opening up possibilities that go beyond human limits.

Today, the market is dominated by one surgical robot: the da Vinci $^{1}$ (Intuitive Surgical, Mountain View, CA, USA) [25]. The system has a high cost: about 2 million $€$ for purchase, $150.000 €$ for annual maintenance and about $1900 €$ of equipment per intervention [66]. It has three components: a console, a patient cart, and a processing unit cart.

The console is where surgeons sit to operate (Figure 1-g,j), they manipulate two joysticks and the system reproduces their motion at the instrument tips (Figure 1-i,h). Each joystick has a sliding button to clutch, so that surgeons can reposition the tips in space without moving the robotic arms. Four pedals activate tools. Surgeons look at the endoscope feed through a stereoscopic display that provides a 3D image of the operating site. The console is meant to provide enhanced ergonomics as it has adjustable arms and head rests, although surgeons have reported neck stiffness, finger, and eye fatigue [36].

The patient cart has three to four robotic arms (depending on the system version) that hold tools and the 3D endoscope (Figure 1-j). The tools are wristed, articulated at the distal end with seven DoF (three orientation, three translation and grip). They reproduce the surgeon's natural wrist movement resulting in increased dexterity, reduction of the path traveled by each hand [47], and increased precision when knot-tying and running sutures [14] when compared to classic MIS. The system can also scale hand motion with a 3:1 or 5:1 reduction.

The third and last component is the processing unit cart, which carries the light source, an insufflator, computing power, and a monitor that displays the endoscope video feed in 2D. The three components add to a considerable footprint. They are usually laid out in the following fashion: the patient cart is placed next to the operating table with the robotic arms going inside the patient through trocars, and the console is placed one or two meters away from the table (Figure 1-f).

The $d a$ Vinci has a system that allows the surgeon to communicate with the surgical team. The console has (1) a microphone that captures the surgeon's voice, which is then reproduced on the processing unit cart, and (2) loudspeakers next to the surgeon's ears where the audio feed from an ambient microphone on the processing unit cart is reproduced. Once surgery starts, the robotic arms are draped and the instruments are inserted into the patient through trocars and attached to the robotic arms. The surgeon sits at the console and the bedside assistant stays next to the patient.

\footnotetext{
${ }^{1}$ In 2014, more than 652.000 surgical interventions were performed using the $d a$ Vinci robot; purchases increased by $12 \%$ from 2014 to 2015 , and predictions indicate a steady growth until 2019 [68]
} 


\begin{tabular}{|c|c|c|c|}
\hline & Classic MIS & Robotic MIS & Interviews \\
\hline S1 & $1 \mathbf{a r}$ & $3+2 \mathbf{a}$ & $2 \dagger$ \\
\hline S2 & $3+3 \boldsymbol{a}$ & $3 \mathbf{a}$ & $3+1 \dagger$ \\
\hline S3 & $1 \mathbf{a}$ & $2 \bullet$ & 1 \\
\hline S4 & & $1+1 \mathbf{a}$ & 2 \\
\hline S5 & & $1 \mathbf{a}$ & 1 \\
\hline S6 & & $2 \boldsymbol{a}$ & 1 \\
\hline N1 / N2 & & & $1 / 1$ \\
\hline I1 / I2 & & & $1 / 1$ \\
\hline Total & $3+5 \mathbf{m}$ & $3+9 \boldsymbol{n}$ & $12+3 \dagger$ \\
\hline
\end{tabular}

Table 1: Study participants. ondicates video-recordings. $\dagger$ Indicates data validation sessions. • Indicates the surgeon was learning. $\mathbf{S}=$ Surgeon. $\mathbf{N}=$ Nurse $. \mathrm{I}=$ Medical Intern.

In HCI, previous work have investigated how surgeons collaboratively construct a mental image of the body [43, 45, 46] and how they control medical equipment [44]. Specifically in robotic-assisted surgery, work has focused on training $[9,28]$, performance metrics $[31,38]$ and identifying future challenges in telesurgery: surgery with distributed medical teams. These challenges include remote intervention planning, awareness of remote activities, mediated communication, and the relation with the remote team and patient given their unfamiliarity with the surgeon $[17,18]$.

Although real, telesurgery remains unrealistic given the technical challenges of keeping a reliable connection open and the need for a specialized team ready on the remote end in case of technical malfunction [16]. For this reason, we investigate the effects surgical robots have on surgical teams when members are co-located. We are not aware of this type of work in HCI, although in the medical community work has shown increased communication [13, 72], which can be seen as an adaptive process [13]. Our work takes a holistic approach, aiming at understanding the effects of the robot on teamwork more broadly.

\section{FIELD STUDY}

We conduct a field study to observe interaction with surgical robots in the wild, and understand how surgeons adopt and adapt this technology. Only through situated studies we can "really understand how [technology] is used, appropriated, and shaped, and in turn how it shapes the work and the lives of the clinicians and patients who interact with it" [6].

\section{Participants}

Table 1 shows the study participants, we worked closely with two gynecology surgeons (S1 and S2) at hospital HA but also with four surgeons in three other institutions to contrast our main observations. All institutions are located in the Paris region, France.

\section{Method}

We first conducted six pilot observations in three institutions in the Paris region, France, to reflect on the study methodology. It became clear that video recordings were necessary, as interaction among people and with technology are rich in details and happen fast and in parallel. We then chose to work closely with two surgeons, performing our main observations, video recordings and interviews with them. We performed a preliminary data analysis and then contacted surgeons from our pilot observations and their colleagues, to perform one day of observations and interviews, contrasting our preliminary analysis.

\section{Data Collection}

Observations and Video Recordings. The surgeon always introduced the researcher(s) to the surgical team and explained the goal of the study, asking them to sign consent forms and media release forms when recording video. We kept field notes during observations, noting the type of intervention and time-stamped notes of interesting events. We did not record any patient data, making sure that the patient was fully covered when recording video. When video recording, we captured 3 video feeds: an overview of the operating room; a GoPro camera strapped to the head of a person next to the patient; and the endoscope.

Interviews. We interviewed members of the surgical team after each intervention when possible, otherwise during the same week. Interviews took place either in an office or at the hospital cafeteria, lasting between $45-75$ minutes. We focused on the differences between classic and robotic-assisted MIS, so we always related our questions to two recent interventions, one of each type. We started by asking interviewees to reflect on events that happened differently in each type of intervention. Some of the mentioned examples were tool switching, teaching interns, surgical strategies, and being in conflict with interns. We probed further by asking details of how each event unfolded for this particular intervention, and how it unfolds in the other type of surgery. We then asked about specific breakdowns when using the robot that we noted during observations, for instance the misunderstanding of an instruction or misactivation of a tool. We asked for details of what had happened, reasons why, and if and how this unfolds without the robot. Likewise for classic MIS, we asked for events that we observed happened differently or did not happen when using the robot, for instance medical interns taking the role of main operator. We finally asked surgeons to reflect on specific moments of past interventions that went especially well (e.g. when a surgery took very little time, when surgeons could operate with minimal or no help) or especially bad (e.g. when they had to convert to open surgery, when the robot failed). 


\section{Data Analysis}

We decided to perform a Thematic Analysis following Braun \& Clarke's [7] methodology because, firstly, we do not intend to discover universal truths as every operating room is unique: they are governed by different laws and institutional practices, shaped by cultural context, with medical staff that has different education, individual experiences, and collective experiences. Also, because we believe that frequency does not determine importance [49], so we did not count occurrences but identified rare and interesting events. We followed a Big $Q$ approach [32] where coding and theme development was a fluid and recursive process. We did not have a defined theoretical positioning, taking an inductive and iterative approach to derive themes purely from the data. Epistemologically, we performed a constructionist analysis: we derived a set of particular and concrete examples of how the introduction of robotics has impacted the work of the surgical team from our observations and interviews; then, through several meetings with surgeons, we constructed together their meaning.

The analysis was performed by one $\mathrm{HCI}$ researcher (western, part of an HCI \& robotics lab focused on surgery and rehab) and two gynecological surgeons (about 8 surgeries a week, 5 years of experience, early and eager tech adopters).

To analyze data, we first listened to all interviews and watched all videos, tagging interesting moments that were different in robotic and classic MIS using ChronoViz [21]. We developed codes to group the events, such as "surgeon asks for help", "communication error", "lack of haptics" and "tool cleaning". Second, we created three diagrams to communicate our initial codes and events to surgeons, putting at the center (1) the surgeon, (2) the team and (3) the technology, then expanding with our codes at the first level and with examples at the second level. We reviewed our findings in three sessions with two surgeons where we discussed possible themes, two lasted two hours and another one hour. In these sessions we generated a new diagram where we constructed meaning around events, identifying for each event (e.g. surgeon grabs the urethra using a piece of fat) its cause (e.g. lack of haptic feedback) and meaning (e.g. perceptual sense shift), from which we derived the final themes. Participants spoke french, we translate quotes into English.

\section{Ethical Considerations}

At the time we conducted this study, our institution did not require ethics clearance as our research 1 ) is not practiced on the human being (ex: testing new medical procedures), neither 2) it aims at developing biological or medical knowledge (biomedical research). HA is a teaching hospital where patients agree to participate in research as part of their treatments.
For the other institutions, participants, or their legal guardians, signed informed consent and a media release form where they stated how we could use their recordings. We did not record any clinical data, nor did we capture video or pictures of patients' faces. Medical staff signed informed consent for the participation of this study, and signed media release forms when recorded, where they stated how we could use their videos. Video recordings, digital copies of notes and interviews were kept in an encrypted hardrive, which only two of the authors can access.

\section{THEMATIC ANALYSIS RESULTS}

We derive one overarching theme that is present in nearly all of our data: the physical separation between surgeons, their teams, and patients impacts the existing practices of surgical teams. We identify three sub-themes: (1) "the autonomous but withdrawn surgeon", (2) "shifting the use of perceptual senses", and (3) "from surgeon assistant to robot assistant".

\section{THE AUTONOMOUS BUT WITHDRAWN SURGEON}

\section{More Control Brings More Autonomy}

Surgical robots open the possibility for controlling multiple tools, each with a high dexterity. The da Vinci in particular allows surgeons to have more control as they can position and lock the endoscope to fix the view, lock a tool to expose the surgical site, and perform surgery with the two remaining robotic tools. This is a radical change from classic MIS where the bedside assistant is in charge of the endoscope and one tool for exposition. Moreover, the high dexterity tools also contribute to autonomy, as they let surgeons reach places by themselves that they could not do before.

Surgeons see the increased autonomy as a unique advantage, as they depend less on the bedside assistant's experience, resulting in a lower variation of the surgical outcome.
"Basically my reflection on robotics is, as much as possible, to diminish the role of the help. So that the influence of the help is as small as possible, so that my surgery is as standardized as possible. So I almost never put helper trocars, I prefer to put three robot trocars rather than two robot trocars and one helper trocar." (S2)

Overloading Surgeon's Cognition. The shift in control increases the cognitive load of an already-burdened surgeon. Two joysticks and six pedals let surgeons control a wide range of functions: moving tools and the endoscope, activating electricity flow on tools, switching to controlling a third arm, modify endoscope focus and changing between normal and fluorescent camera. To accommodate for all these functions, the console uses modes that map the joysticks and 
pedals to different functions. Unsurprisingly, modes lead to errors and sometimes risky situations.

The first mode error we observed lead to moving tools when intending to move the endoscope, creating the risk of piercing through tissue. In both functions the surgeon manipulates the joysticks, but for the endoscope the surgeon must push at the same time the camera pedal. We observed that surgeons were quick enough to realize that the result was not what they expected, so they immediately stopped their hand motion and restarted the action by first stepping on the pedal.

The second was unintendedly activating the fluorometric camera, this time by pushing the camera pedal at the same time as the joystick clutch buttons. The view then becomes black as there is no fluorescent dye and surgeons shortly move the tools blindly, which can be enough to unintendely damage a structure. Though we are not sure of the exact reason behind this mode error, we suspect that surgeon try to move the endoscope, thus push the camera pedal, but without having yet let go of the clutch buttons from repositioning the joysticks.

Thirdly, we observed instances where surgeons pushed the wrong pedal and thus activated the wrong tool. Video analysis showed that this happened mostly when the surgeon had just sat at the console, or when the assistant had just switched tools-thus inverting them. We believe the confusion comes from the different mental models of how pedals work. In classic MIS there are two separated pedals, each one connected to one tool, which surgeons can spatially arrange as they wish, associating a foot pedal with a type of operation, not with the hand holding the tool. The da Vinci however associates the right (left) pedal with the tool controlled by the right (left) joytstick, regardless of the operation performed by the tool.

To avoid these mode activation errors, surgeons adapt their surgical strategy, which can overload their cognition.

"I take the tissue that I want to coagulate, and I always have my [other] hand that does not touch anything, in case I confuse the pedals. Because if I ever do not see it and it touches the intestine, and I [makes buzz sound] it makes a hole in the intestine, so, by reflex, and S2 too, now, we always learn to have both instruments visible, so that if do a mistake, it does not matter.” (S1)

\section{A Withdrawn Surgeon}

The console centralizes control and thus the surgeons' autonomy, but siting behind the console withdraws the surgeon from the team, both physically and socially.

"Out of (Surgical) Site, out of Mind". It came as a surprise to us that in almost every intervention, once surgeons sit at the console, they become out of the surgical team's sight, and out of their minds. In classic MIS, when the surgeon stands on the patient's side, people are ready to assist: medical interns ask questions, make suggestions and operate tools; and, nurses foresee and prepare the tools and materials the surgeon will needed next. In robotic-assisted MIS however, team members have a lessened perception of the surgeons' presence acting as if they were not in the room. Conversation about the weekend dominates the dialog and non-sterile staff check their phones, impacting the team's ability to anticipate the surgeons' needs and also annoying them.

"The worst part of [the robot], is that because the
surgeon is stuck at the end of the room, people
come and go, talk, and consider that as the surgeon
is not in front of them, well, they can talk. [..] for
an intervention of five hours, in the end it's painful,
as you are focused and you hear three different
conversations of the weekend, tomorrow's shifts,
and the program of the OR next door. It's just
unbearable." (S6)

Lower Situational Awareness. Situational awareness is particularly important as lack thereof can lead to medical errors [24]. In classic MIS, we observed that team members naturally observe each other's actions even when these are only visible through the corner of their eyes, they listen to conversations, they interpret sounds like tools falling on the floor, they identify smells like burnt tissue, and they feel each other's movement through hands and tools. This multisensory information provides a constant picture of other people's activities. A teleoperated robotic system in contrast results in surgeons immersed in the console and isolated from the team. The lack of haptics and limited visual contact with the team, which is discussed in detail in the next theme, decrease the surgeon's awareness of events that happen at the patient side.

When instructing assistants, surgeons cannot visually verify if their instructions were understood, if they are being executed or if they are purposely delayed. Surgeons can only become aware of this either by explicitly asking for it or by observing changes through the endoscope (e.g. assistant tool movement).

The lack of awareness is also present in actions not initiated by the surgeon, such as an alert from medical equipments. We observed during one intervention that the insufflation machine was running low on $\mathrm{C} 02$ gas and thus started beeping. S2 was not aware whether someone initiated the bottle change, so he asked: "<Circulating nurse name>..? Yes? - It beeped.. - That's right, that's what I was doing, I was preparing to change the bottle". Surgeons in general thought that having to ask for confirmation and about details of event at the bedside was annoying and interrupted them. 
Finally, situational awareness about the robot itself is also hard to accrue. S6 noticed in an interview while looking at an intervention that the endoscopic arm of the $d a$ Vinci did a circular motion and looped the camera cable around an arm.

"See that? That is really dangerous. If I had moved the camera, it would have pulled and moved that arm, then the tool can hit anything inside." (S6)

Socially Withdrawn. We found that the gain in control comes at the price of isolation both professionally and personally. S1 and S2 recognized during interviews that they discuss less with the medical team about the current surgery. In contrast, we observed in classic MIS that surgeons constantly verbalized their strategy, saying out loud what they are doing and why. This puts all the team on the same page, assistants can comment on the strategy and learn from it, and instrumentalist can anticipate tool needed next. Surgeons also discuss less with bedside assistants and nurses, if at all, about personal life. Although the $d a$ Vinci has a communication system, all surgeons perceive it mostly as a mean to provide instructions and not for private conversations, as there is no privacy when talking through the loudspeakers.

$$
\text { "It's harder to get to know the interns." (S6) }
$$

\section{SHIFTING THE USE OF PERCEPTUAL SENSES}

Current teleoperated surgical robots impair surgeons' use of perceptual senses by removing haptics and limiting visual contact with their teams and patients. Surgeons in our study coped by shifting which senses they used to perceive information, creating new conflicts with the bedside assistant.

\section{Lack of Haptic Feedback}

The da Vinci console does not provide haptic feedback to the surgeon's hands, a radical change from classic MIS where surgeons rely on touch to handle tissue by feeling its stiffness and evaluating how far they can pull without breaking it.

\section{"I touch with my eyes." (S1)}

All the surgeons in our study reported that they can replace haptics with 3D vision, as they learn to interpret tissue deformation. However, we observed that this adaptation strategy does not let surgeons feel actions outside their view.

Replacing Haptic with Visual Feedback. Finding tools outside the endoscope's field of view cannot be performed through touch. We observed in classic MIS that surgeons know the approximate position of their tools through proprioception, and they can move them towards the endoscope using haptic feedback as a guide. Although they use long tools, surgeons can still determine object features like shape, texture and consistency [5], and can guide their movements without looking. The lack of haptics and proprioception in robotic-assisted
MIS means that tool motion has to be checked visually, otherwise surgeons risk breaking tissue without feeling it. Thus, surgeons move the endoscope towards the tool instead of the tool towards the endoscope, decreasing the chance of unintendedly damaging tissue. We observed however occasions where surgeons moved tools into the field of view only to find that they were pulling on tissue stuck at the tool tip. This rarely, if ever, happens in classic MIS as surgeons' movements are supple and they can reduce the force or stop moving when they notice pulling on a tissue.

Another issue occurs when tools are blocking each other outside the field of view. In classic MIS, surgeons feel conflicts through the haptic sensations transmitted by tools, and can sometimes re position them to solve the conflict without necessarily moving the endoscope to look. In robotic-assisted MIS, surgeons only detect conflicts when they observe unexpected behaviors through the endoscope, e.g. when a tool does not respond to joystick movement as it is blocked by another tool, or when the endoscopic view moves as it is pushed by a moving tool. Surgeons have no other choice but to move the endoscope and find the conflict to re-position the tools.

\section{S1: "I miss it [haptic feedback] when I have lost my instrument, to know where it is, to avoid .." Interviewer: "Instead of having to find it with the camera, to find it with your ..?" S1: "with touch of course!"}

The visual channel is thus overloaded as it has to be used for surgery (primary) and for solving conflicts (secondary task).

Replacing Haptic with Verbal Feedback. In classic MIS, surgeons can demonstrate actions by grabbing the assistant's hands and performing the movement. As surgeons now sit at the console, far form the assistant, they have no choice but to use lengthy verbal instructions.

"I wish I could control the intern with the robot."

(S4)

Workarounds When Haptics Cannot Be Replaced. Handing delicate tissue without damaging itwith the medical team. heavily relies on touch. S1 and S2 explained how they change their surgical strategy as 3D vision is not enough to replace touch. Instead of squeezing when handling the urethrea or the intestines, they either push or grab this delicate tissue from an adjacent structure, such as a piece of fat, to avoid accidental tearing. In extreme cases, the lack of haptics can lead to converting to open surgery. For instance, S5 explained that he needed to select a segment of intestine that could be pulled into a new position without tearing an attached vein for vascularization. He tested many segments by pulling with the robotic arms, but "feeling" tension through vision 
was not enough to determine if the vein would break. He had no choice but to convert to open surgery and feel tissue tension with his hands. Converting to open surgery has a major impact, as it has all of the overheads of using the robot (cost, preparation, time) and none of the benefits for patients as a large incision is performed.

Adapting the endoscope to use it as a surgical tool, beyond its use as a camera, is a practice that relies on haptics. We observed during para-aortic lymph node dissection in classic MIS that two surgeons (S1 and S2) sometimes dissect tissue using the endoscope. They break through tissue with the endoscope shaft, as they can rely on haptics to guide their actions. This came as a surprise as dissection is normally performed using bipolar forceps. But in robotic-assisted MIS, surgeons admitted that they do not do this anymore as there are no haptics, and pushing the camera into tissue would occlude the view, effectively operating with no feedback whatsoever.

Cleaning instruments is also affected by the loss of haptics. In classic MIS, surgeons can clean instruments either inside the patient by rubbing them against each other or outside the patient using a compress (gauze). In robotic-assisted MIS however, cleaning instruments inside the patient can break them as surgeons cannot feel how hard they rub them against each other. This means that they have to take tools out, which takes longer than in classic MIS.

\section{Limited Visual Contact with Team}

Surgeons have a limited view of the surgical field during robotic surgery as they are immersed in the stereoscopic view. Although they say that this is not a problem, we observed several ways in which they adapt their behavior to this limitation.

Replacing Visual With Verbal Feedback. The lack of line of sight in robotic-assisted MIS increases task-related communicative acts as non-verbal cues have to be made explicit $[18,33]$. A more detailed analysis using a hierarchical task decomposition in previous work [8] found that with the surgical robot, surgeons require more information and new information of different types, mainly from the robotic arms, as they do not have direct visual access to them. This increase is an adaptive process [54] as more information needs to be exchanged verbally about the instrument orientation and placement, instructions and confirmations need to be made explicit, and even proprioceptive feedback becomes verbal as surgeons ask nurses to feel tissue. Scripting speech can mitigate the increase in verbal communication [72], which establishes common ground among team members [10], although this approach is rarely adopted in real settings.

On top of increasing verbal exchanges, these also become more difficult. Surgeons oftentimes remove their heads from the console to talk directly to their medical teams, as it is often the case when there is noise in the room and the communication system is turned off, loosing focus [18, 33].

In our study, we observed instances when verbal communication replaced missing visual cues. Surgeons had to verbally describe actions to assistants that before were demonstrated with a simple gesture, such as a rotation direction. Moreover, verbal descriptions oftentimes did not suffice, so surgeons stood up from the console, walked to the surgical field and talked face to face. In extreme cases, surgeons scrubbed in and performed actions themselves to get them done and to continue operating, but also to teach assistants.

S2's monopolar scissors does not coagulate anymore. "It doesn't work anymore, can you please plug it back again?" S3 is at the second console, retries, still no electricity flow. S2 walks to the surgical field and explains using hand gestures how to plug the cable correctly.

We probed surgeons further to understand why verbal communication is hindered, even with a communication system in place. Surgeons explained that the field microphone is oftentimes far from the assistant and it barely captures their voices, so they have to repeat themselves or yell. Moreover, they mentioned that the field microphone also captures parallel conversations, so they turn it off to avoid distractions. Lastly, they explained how the high level of noise from the equipment made it hard to talk and listen from the distant console, and how they sometimes remove their heads from the stereoscopic display to communicate, which interrupts their flow.

All in all, we find that perception is both under and over loaded: the underloaded sense of touch overloads the visual sense as it is used for primary and secondary tasks. Lastly, the hearing sense is overloaded as it replaces haptics and visuals.

\section{New Communication Conflicts}

Instructing assistants in classic MIS is a fluid process, as surgeons can adapt their strategy just from looking at the state of the operating table and at the assistants' actions. In robotic-assisted MIS however, as we have discussed, several factors impact communication, including the increase in verbal communication to replace implicit communication, increased misunderstandings, and the higher level of noise from the robot and from parallel conversations.

Communication conflicts can impact work as communication plays a critical role in the OR. Communication is a key factor for good collaboration and a successful and safe surgery $[60,64,71]$, failures have been shown to increase cognitive load, interrupt routines, and increase tension, ultimately affecting surgical outcome [37]. A meta review 
of teamwork in health care [40] shows evidence that the staff's perception of teamwork affect quality and safety of patient care, and that communication, coordination, and leadership support effective teamwork. In robotic-assisted MIS, poor communication has been correlated to higher estimated blood loss and longer operative times, mainly due to the high noise and console-to-bedside communication issues [61].

We observed in our study that actions inside the patient have a lower coordination because of the already discussed lack of haptics. Surgeons can block the assistant's tools and not realize so because of collisions outside the field of view, similarly to how it occurs with their own tools. For instance, we observed situations in gynecology where the surgeon asks the assistant to move the uterine manipulator, but many times the assistant would report that he's pushing to the maximum though in reality his movement was blocked by one of the robotic arms. The surgeon thus has to move the endoscope, interrupting his current task, to help the assistant perform the action.

Moreover, we observed that actions outside the patient also had have a lower coordination, this time from the limited visual contact. Surgeons' actions become unpredictable robot arm motions from the assistant's perspective, as small motions at the tool tip translate into large motions at the back, because of the lever effect created from inserting tools through small holes. As a consequence, the robotic arms are sometimes on the assistants' way. We observed in two occasions interns being violently hit by an arm and asking the surgeon to stop the surgery as they catch their breath.

S3 sits at the console while S2 instructs him. The intern is inserting a tool into one the robotic arms. S3 moves a second tool and hits the intern. Intern: "Sh*t". S2: "Where is it?" Intern: "Could you please stop moving? You just hit me in the breast and it hurts."

Communication misunderstandings became a recurrent observation in our study. It was common for assistants to start executing an instruction and have the surgeon stop and correct them, as a misunderstanding put them in conflict. For example during a tool change, the surgeon would ask to remove a tool by its type (e.g. monopolar scissor) or side (e.g. the left tool), but because the assistant does not manipulate the same tools as the surgeon, she does not always have a clear notion of which tool is attached to which arm. As a result, she would start removing the wrong tool, the surgeon would notice, tell her to stop and re attach the tool, and finally to start over with the next robotic arm.

\section{FROM SURGEON ASSISTANT TO ROBOT ASSISTANT}

It became clear during our study that surgeons become their assistants during robotic surgery, as they assist their own surgical gestures by controlling multiple arms. The bedside assistant role then suffers a profound change as a consequence: from assisting the surgeon to assisting the robot.

\section{Assistants Perform Fewer Surgical Gestures}

Surgeons rely less on assistants both because they can perform more surgical gestures autonomously, and because they avoid asking for help as this interrupts their flow.

First, as surgeons control more robotic arms, assistants perform fewer gestures. When the robot has three arms, the assistant is responsible for manipulating laparoscopic instruments and doing hemostasis [65]. A fourth arm enables surgeons to perform all these gestures, reducing their reliance on highly trained assistants $[19,59,67]$. The only surgical actions assistants perform are those that the surgeon cannot do from the console, such as lavage and aspirating fluids, inserting objects into the patient (e.g. a needle or endobag), removing objects (e.g. a needle or specimen extraction), and clipping [74]. We observed in our study this same decline in assistants' surgical gestures, starting with the fact that assistants manipulated one laparoscopic tool instead of two.

\section{"A tool switch means I'm forced to take a break."}

Second, S1 \& S2 explained that they avoid asking for help as this interrupts their flow. The difficulties in communication we have already discussed in Theme 2 lead surgeons to strive before resigning and asking for help, whereas in classic MIS they tend to ask for assistance much sooner. Specifically, we observed that surgeons avoid asking for help during bleeding and tool swaps. When bleeding occurs, the assistant provides suction to make the operative site visible. We observed systematic misunderstandings regarding the exact place of suction and for how long, which lead surgeons to take extra precautions that avoid bleeding, even if small, to avoid asking for assistance. Tool switching is sometimes necessary to increase comfort, as it can improve the angle from which tools approach tissue. We observed situations where surgeons strove as much as they could before asking assistants to switch tools, because this process takes much longer than in classic MIS.

"The less you make bleed, the less help you need."

(S2) 
From Learning by Doing To Learning by Watching. Surgery is not only for improving patients' health, but also to teach medical interns. We observed that the classical mentoring model of Bedside Teaching radically changes from a multi-sensory experience when performing gestures next to a supervising surgeon to passively watching the endoscope video. Interns do not get to perform surgical gestures themselves anymore, they neither observe surgeons demonstrating how to manipulate tools, nor they learn by having the expert surgeon take their hands and guide them through a gesture. Some surgeons and medical interns in our study believe that medical students learn less about surgery.

"They [interns] are not focused, they don't follow, communication is hard, so they don't learn anything." (S2)

One interesting comment came from I1 during an interview, where she explained why this change is an opportunity to learn differently: being less involved means that she has the time to watch the endoscope video with fewer interruptions. She can learn about the surgeon's long-term strategy, rather than focusing on learning individual gestures.

Learning Surgical Gestures Through The Console. To learn how to perform surgical gestures in robotic surgery, interns would need to leave the bedside, sit at the console and take over control of the robotic arms. We observed mentoring on the console from S1 and S2 towards S3. We realized that mentoring radically changes from the multi-sensory experience of classic MIS: the teaching surgeon cannot grab the learner's hands and guide their movements on the joysticks, as this is dangerous because the teacher does not see the endoscopic feed. Surgeons cannot teach by demonstration either, as having a learner look at a surgeon controlling joysticks does not provide information about a surgical gesture. So learners can only receive verbal instructions from surgeons that stand behind them or sit at a second console. A 3D virtual cursor can be drawn on the stereoscopic view to point, though surgeons reported that the virtual pointer is not a great tool for teaching, as it is not expressive enough to convey meaning.

All in all, there is large room for improving skill acquisition on the console; perceptual senses can be further improved, for instance by adding haptic feedback, which has been shown to improve learning in a teleoperated console [30], and simulators can be used for teaching [62].

\section{Assistants Perform Robotic Support}

Previous work has shown that bedside assistants are in charge of new tasks related to the robot, mainly helping with dressing and docking before the surgery, changing tools and untangling arms in case of conflicts during surgery, and helping with robot retraction after surgery $[42,63]$. They also have the new task of reporting on any noticeable patient complication as the surgeon is away, which requires observational and communication skills [63]. Assistants' experience in robotic surgery is of special importance, as it has been correlated with lower intervention times [51] and lower estimated blood loss [29].

In our study, we observed several factors that lead to assistants switching from assisting surgeons to assisting robots: robotic surgery is not taught in school, so first year medical interns cannot operate, interns change every six months, which is not enough time to teach how to use the robot, and, the structure of the hospitals we observed do not have other personnel that can take the role of "robotic" bedside assistants, so assistants are tied to that position. We probed surgeons with the question of having someone other than a medical intern as the bedside assistant, but they explained that currently it is not possible given their institution's structure. They could envision nurses in that role as long as (1) they are Registered Nurse First Assistants ${ }^{2}$ (RNDFA), so they can perform intraoperative gestures under their supervision and (2) that they also receive specialized training for the robot. The reality however is that there are few RNDFA nurses in their departments, which are assigned to surgeries by prioritizing the general department's schedule, so they cannot be guaranteed in robotic surgery. Furthermore, there is no specialized course for nurses about managing the robot. So today the solution is to keep the medical interns as bedside assistants, even if their potential is underused.

It seems then that there is place for a new role of "robotic bedside assistant", which would need to be fulfilled by someone with specialized training on manipulating the robot and the robotic tools. Learning these skills "on the job", similar to how surgical skills are learned, is hard [42], pointing to the need for specialized training before going into the operating room. Bedside assistants in our study however reported learning to assist the robot through practice and not through specialized training. This shows that the adoption of the surgical robot can lead to a disconnect between defined procedures and reality.

\begin{abstract}
"You have to know how to manipulate the arms, there is a way to do it, you have to know how to interpret the colors. For example last week, [..] I had a problem on one arm, [..] so I called the company da Vinci to find out what happened because the robot is permanently connected, the customer service actually sees what happens. So I called and they said there was a power supply problem I do not know why." (N1)
\end{abstract}

\footnotetext{
${ }^{2}$ https://nurse.org/resources/rnfa-career-guide/
} 
Lastly, we noted that the new role of the assistant was less demanding than in classic MIS, as we observed assistants spending long periods of time, sometimes of even an hour, with no assigned task. As a consequence of their idleness, bedside assistants tend to be more distracted, talking to colleagues about non-task related topics and paying less attention to the endoscope video feed. This behavior impacts their ability to foresee a surgeon's needs and to understand instructions.

\section{STUDY LIMITATIONS AND FUTURE WORK}

Our study included a limited population of medical staff, in only three institutions and one city. After observing several surgical teams, it became clear to us that these specialized workers are unique and extremely diverse, so other surgical teams will undergo different impacts of the same technology. We would like to see more studies similar to ours, so that our findings can be compared, contrasted and even challenged.

We aimed at a holistic view of the impacts of the technology, identifying and characterizing the richness of the interaction with the technology from various perspectives such as human perception, team work, communication and skill acquisition. We did not intended to quantify occurrences of particular events to find causal relations with, say, errors or surgeon and team experience. Future work can use our observations as a basis for more focused, perhaps quantitative, studies, that can determine the exact causal relation of the observed impacts.

Lastly, we would like to inspire work that brings the fields of $\mathrm{HCI}$ and robotics closer together, so that future surgical robots have both excellent engineering capabilities and also integrate seamlessly with the work of surgical teams.

\section{DISCUSSION}

Our study has confirmed previous observations and expanded on further unintended and unexpected consequences of roboticassisted MIS. We believe these changes are due to the fact that the telemanipulated approach separates surgeons from their teams and patients. We question the telemanipulation approach: are there other ways for designing surgical robots?

To answer this, we first discuss the telemanipulated approach origin, we revisit our findings of current practices, and finally we discuss a new dimension for the design of future systems.

\section{Past: How Did We Get to Telemanipulation?}

We draw from Lanfranco et al.'s [35] detailed history of robotic surgery. Development started in the 80's with the use of commercially available telemanipulated robotic arms for specific procedures. Two giants then became interested in the possible applications of remote surgeries: NASA, to operate on astronauts in space, and the US Army, to bring surgery closer to the battle field. Surgeons and engineers noticed the potential for these articulated robots to overcome the limitations of classic MIS as they could restore the lost dexterity. Then, the US Army provided funding for private development of telemanipulated surgical robots, which ultimately lead to the da Vinci today. This brief summary suggests that telemanipulation is a design choice to achieve teleoperation, rather than a necessary condition to overcome the shortcomings of MIS.

\section{Present: Telemanipulation Today}

Our study reflects the increasing adoption rates of the $d a$ Vinci robot: all the surgeons were highly satisfied with the robot particularly appreciating the high dexterity and 3D vision, with their institutions having plans to expand surgical robot programs. But our study also shows that the undesirable impacts of robotic surgery are widespread: perceptual senses are impoverished, communication is hindered, surgeons and assistants' roles change and knowledge acquisition is radically affected. These impacts and the elevated financial costs raise the question: why is the technology so widely adopted?

First, the impacts we observed vary from surgeon to surgeon. Our observations of S6 points towards the possibility of mitigating some of these impacts with time. S6 is a surgeon with considerable experience, being recognized by her peers as a leader in robotic surgery. During two observations, she operated with a medical intern with whom she had been working together for just over six months. We were impressed by the speed and accuracy in which she performed surgery, puting our previous observations in perspective. Coordination was high; surgeon and assistant acted upon the same tissue rarely if ever in each other's way, with few communication misunderstandings. Given that experience and good teamwork improves surgical outcome, is it possible that clinical benefits for patients will appear when surgeons master the robot?

Another important reason for adoption is to bridge the gap between open surgery and minimally invasive procedures. MIS adoption is limited by the skills needed to master the technique, such as video-hand coordination, depth perception and lack of dexterity for suturing [23]. The European Association for Endoscopic Surgery adopted in 1995 the consensus to use minimally invasive procedures as the standard for all interventions where there are no particular contraindications [52]. But in regions where MIS is not highly developed, robotic surgery has made the jump from open to minimally invasive procedures. In these cases, the benefits of MIS are attributed to robotic MIS, a bias that is largely exploited during marketing. 


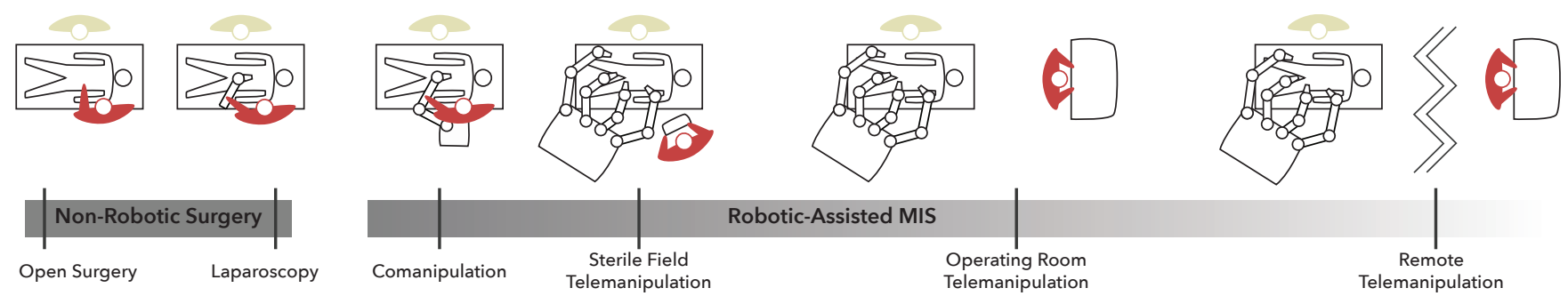

Figure 2: Exploring the surgeon-patient design dimension for robotic-assisted MIS.

\section{Future: Exploring the Surgeon-Patient Distance and New Opportunities for $\mathrm{HCl}$}

We discuss in this section the design dimension surgeonpatient distance for future surgical robots, and we present new opportunities for $\mathrm{HCI}$ that this dimension brings.

We see the surgeon-patient distance as a continuum (Figure 2): Telemanipulation fills most of it, with various degrees of surgeons-patient distance, and Comanipulation, as defined by Morel et al. [48], lies at the left of the continuum.

Remote Telemanipulation lies on the far right of the continuum, where the surgeon is not in the operating room [41]. This setup makes sense when the distance is imposed by external factors.

Operating Room Teleoperation lies as we move towards the left, where surgeons are co-located but still segregated from their teams, as it is the case of the $d a$ Vinci. An opportunity for $\mathrm{HCI}$ is to improve audio, visual contact and haptics so that surgeons can better interact with their teams. Audio: Team members could wear microphones that capture their voices, which are then reproduced on the console. Presenting multiple audio sources needs careful consideration, as S4 explained, the tight space for the head on the console currently echoes other peoples' conversations which distracts him. Video: video of the surgical field could be captured using a mounted camera and presented in the surgeons' view. To avoid creating distractions that potentially lead to safety concerns, surgeons or medical staff could have control of this video's visibility, perhaps also tracking surgeons' gaze to limit tool movement when they are not looking at the endoscope. Haptics: we are aware that implementing haptics on telemanipulators is a complex engineering problem, surgeons in our study reported that Intuitive Inc. has been pursuing this for long time, although there is no planned release to the market of this feature.

Sterile Field Telemanipulation lies further to the left, here $\mathrm{HCI}$ can study the interaction design with a smaller, sterilized and portable console, so that surgeons can teleoperate while in the sterile field. Stereoscopic vision does not need to be sacrificed in this setup, as it can be provided by 3D screens, which are used today in surgical rooms. A console on the sterile field would improve communication and perhaps both the surgeon and assistant could manipulate one console each to control four or more robotic arms in coordination.

Comanipulation lies at the far left of the continuum, where surgeons, or assistants, manipulate laparoscopic tools along with robots. In this scenario, surgeons would have a clear view of their teams, patient, and have haptic feedback when using mechanical tools. We believe one upcoming opportunity for $\mathrm{HCI}$ is the design of natural interfaces with comanipulated tools that have high degrees of freedom, such that the surgeon and the system become partners: one provides the intelligence for decision making and the other the ability to do precise and repeated movement. Teleoperations could still be achieved through comanipulation, for instance the surgeon could operate on a "ghost" of a remote patient, which is then translated into motion of a surgeon's robot avatar in the remote location. Augmented reality can also provide an immersive experience to bring surgeons closer to their teams.

\section{ACKNOWLEDGMENTS}

This research was supported by the Labex CAMI (project ANR-11-LABX-0004) operated by the ANR as part of the program "Investissement d'Avenir". We thank all the participants of this study for their valuable time, and Susanne Bødker, Joanna McGrenere and Emeline Brulé for their insights during data analysis.

\section{REFERENCES}

[1] J. D. F. Allendorf, M. Bessler, R. L. Whelan, M. Trokel, D. A. Laird, M. B. Terry, and M. R. Treat. 1997. Postoperative immune function varies inversely with the degree of surgical trauma in a murine model. Surgical Endoscopy 11, 5 (May 1997), 427-430. https://doi.org/10.1007/ s004649900383

[2] Joan S. Ash, Marc Berg, and Enrico Coiera. 2004. Some Unintended Consequences of Information Technology in Health Care: The Nature of Patient Care Information System-related Errors. Fournal of the American Medical Informatics Association 11, 2 (March 2004), 104-112. https://doi.org/10.1197/jamia.M1471 
[3] R. Berguer. 1998. Surgical technology and the ergonomics of laparoscopic instruments. Surgical Endoscopy 12, 5 (May 1998), 458-462. https://doi.org/10.1007/s004649900705

[4] R. Berguer, D. L. Forkey, and W. D. Smith. 1999. Ergonomic problems associated with laparoscopic surgery. Surgical Endoscopy 13, 5 (May 1999), 466-468. https://doi.org/10.1007/PL00009635

[5] Omar S. Bholat, Randy S. Haluck, Willie B. Murray, Paul J. Gorman, and Thomas M. Krummel. 1999. Tactile feedback is present during minimally invasive surgery11US Surgical provides a grant to Penn State for training in laparoscopic surgery. A portion of the Penn State salary for Dr Omar Bholat is from the US Surgical grant. Funds from the grant are used to support research activity in Dr BholatâĂŹs laboratory. Fournal of the American College of Surgeons 189, 4 (Oct. 1999), 349-355. https://doi.org/10.1016/S1072-7515(99)00184-2

[6] Ann Blandford, Erik Berndt, Ken Catchpole, Dominic Furniss, Astrid Mayer, Helena Mentis, Aisling Ann OâĂŹKane, Tom Owen, Atish Rajkomar, and Rebecca Randell. 2015. Strategies for conducting situated studies of technology use in hospitals. Cognition, Technology \& Work 17, 4 (Nov. 2015), 489-502. https://doi.org/10.1007/s10111-014-0318-7

[7] Virginia Braun and Victoria Clarke. 2006. Using thematic analysis in psychology. Qualitative Research in Psychology 3, 2 (Jan. 2006), 77-101. https://doi.org/10.1191/1478088706qp063oa

[8] Caroline G. Cao and Holly Taylor. 2004. Effects of New Technology on the Operating Room Team. Technical Report. Tufts University, Dept Og Mechanical Engineering. http://www.dtic.mil/docs/citations/ ADA438402

[9] Nicklas H. Christensen, Oliver G. Hjermitslev, Frederik Falk, Marco B. Madsen, Frederik H. ÃŸstergaard, Martin Kibsgaard, Martin Kraus, Johan Poulsen, and Jane Petersson. 2017. Depth Cues in Augmented Reality for Training of Robot-assisted Minimally Invasive Surgery. In Proceedings of the 21st International Academic Mindtrek Conference (AcademicMindtrek '17). ACM, New York, NY, USA, 120-126. https: //doi.org/10.1145/3131085.3131123

[10] Herbert H. Clark and Susan E. Brennan. 1991. Grounding in communication. In Perspectives on socially shared cognition, L. B. Resnick, J. M. Levine, and S. D. Teasley (Eds.). American Psychological Association, Washington, DC, US, 127-149.

[11] R. I. Cook and D. D. Woods. 1996. Adapting to new technology in the operating room. Human Factors 38, 4 (Dec. 1996), 593-613. https: //doi.org/10.1518/001872096778827224

[12] R. I. Cook and D. D. Woods. 1996. Implications of automation surprises in aviation for the future of total intravenous anesthesia (TIVA). fournal of Clinical Anesthesia 8, 3 Suppl (May 1996), 29S-37S.

[13] S. Cunningham, A. Chellali, I. Jaffre, J. Classe, and C. G. L. Cao. 2013. Effects of Experience and Workplace Culture in Human-Robot Team Interaction in Robotic Surgery: A Case Study. International fournal of Social Robotics 5, 1 (Jan. 2013), 75-88. https://doi.org/10.1007/ s12369-012-0170-y

[14] G. F. Dakin and M. Gagner. 2003. Comparison of laparoscopic skills performance between standard instruments and two surgical robotic systems. Surgical Endoscopy And Other Interventional Techniques 17, 4 (April 2003), 574-579. https://doi.org/10.1007/s00464-002-8938-z

[15] Chad J. Davis and Charles J. Filipi. 1995. A History of Endoscopic Surgery. In Principles of Laparoscopic Surgery: Basic and Advanced Techniques, Maurice E. Arregui, Robert J. Fitzgibbons, Namir Katkhouda, J. Barry McKernan, and Harry Reich (Eds.). Springer New York, New York, NY, 3-20. https://doi.org/10.1007/978-1-4612-2480-8_1

[16] Charles R. Doarn and Gerald R. Moses. 2011. Overcoming Barriers to Wider Adoption of Mobile Telerobotic Surgery: Engineering, Clinical and Business Challenges. In Surgical Robotics. Springer, Boston, MA, New York, NY, USA, 69-102. https://doi.org/10.1007/
978-1-4419-1126-1_4

[17] Pieter Duysburgh, Shirley A. Elprama, and An Jacobs. 2014. Exploring the Social-technological Gap in Telesurgery: Collaboration Within Distributed or Teams. In Proceedings of the 17th ACM Conference on Computer Supported Cooperative Work \& Social Computing (CSCW '14). ACM, New York, NY, USA, 1537-1548. https://doi.org/10.1145/ 2531602.2531717

[18] Shirley A. Elprama, Katriina Kilpi, Pieter Duysburgh, An Jacobs, Lotte Vermeulen, and Jan van Looy. 2013. Identifying Barriers in Telesurgery by Studying Current Team Practices in Robot-assisted Surgery. In Proceedings of the 7th International Conference on Pervasive Computing Technologies for Healthcare. ICST (Institute for Computer Sciences, Social-Informatics and Telecommunications Engineering), ICST, Brussels, Belgium, Belgium, 224-231. https://doi.org/10.4108/icst. pervasivehealth.2013.252005

[19] Michael P. Esposito, Pedram Ilbeigi, Mutahar Ahmed, and Vincent Lanteri. 2005. Use of fourth arm in da Vinci robot-assisted extraperitoneal laparoscopic prostatectomy: novel technique. Urology 66, 3 (Sept. 2005), 649-652. https://doi.org/10.1016/j.urology.2005.03.061

[20] Fernando Flores, Michael Graves, Brad Hartfield, and Terry Winograd. 1988. Computer Systems and the Design of Organizational Interaction. ACM Trans. Inf. Syst. 6, 2 (April 1988), 153-172. https://doi.org/10. $1145 / 45941.45943$

[21] Adam Fouse, Nadir Weibel, Edwin Hutchins, and James D. Hollan. 2011. ChronoViz: A System for Supporting Navigation of Time-coded Data. In CHI '11 Extended Abstracts on Human Factors in Computing Systems (CHI EA '11). ACM, New York, NY, USA, 299-304. https: //doi.org/10.1145/1979742.1979706

[22] K. H. Fuchs. 2002. Minimally invasive surgery. Endoscopy 34, 2 (Feb. 2002), 154-159. https://doi.org/10.1055/s-2002-19857

[23] Noga Fuchs Weizman, Rie Maurer, Jon I. Einarsson, Allison F. Vitonis, and Sarah L. Cohen. 2015. Survey on Barriers to Adoption of Laparoscopic Surgery. Fournal of Surgical Education 72, 5 (Oct. 2015), 985-994. https://doi.org/10.1016/j.jsurg.2015.04.001

[24] M. Graafland, J. M. C. Schraagen, M. A. Boermeester, W. A. Bemelman, and M. P. Schijven. 2015. Training situational awareness to reduce surgical errors in the operating room. BJS 102, 1 (Jan. 2015), 16-23. https://doi.org/10.1002/bjs. 9643

[25] G. S. Guthart and J. K. Salisbury. 2000. The Intuitive(TM) telesurgery system: overview and application. In Proceedings 2000 ICRA. Millennium Conference. IEEE International Conference on Robotics and Automation. Symposia Proceedings (Cat. No.00CH37065), Vol. 1. IEEE, San Francisco, CA, USA, 618-621 vol.1. https://doi.org/10.1109/ROBOT. 2000.844121

[26] C. N. Gutt, T. Oniu, A. Mehrabi, A. Kashfi, P. Schemmer, and M. W. BÃijchler. 2004. Robot-assisted abdominal surgery. BfS 91, 11 (2004), 1390-1397. https://doi.org/10.1002/bjs.4700

[27] J. G. Holden, J. M. Flach, and Y. Donchin. 1999. Perceptual-motor coordination in an endoscopic surgery simulation. Surgical Endoscopy 13, 2 (Feb. 1999), 127-132. https://doi.org/10.1007/s004649900920

[28] Yu-Hsuan Huang, Hao-Yu Chang, Wan-ling Yang, Yu-Kai Chiu, TzuChieh Yu, Pei-Hsuan Tsai, and Ming Ouhyoung. 2018. CatAR: A Novel Stereoscopic Augmented Reality Cataract Surgery Training System with Dexterous Instruments Tracking Technology. In Proceedings of the 2018 CHI Conference on Human Factors in Computing Systems (CHI '18). ACM, New York, NY, USA, 465:1-465:12. https://doi.org/10.1145/ 3173574.3174039

[29] Ilana Jacobs, Aaron Lay, and Jeffrey Gahan. 2015. MP23-17 LACK OF AN EXPERIENCED BEDSIDE ASSISTANT MAY ADVERSELY AFFECT OUTCOMES DURING ROBOTIC PROSTATECTOMY. The fournal of Urology 193, 4 (April 2015), e273-e274. https://doi.org/10.1016/j.juro. 2015.02.1260 
[30] Stephan Jacobs, David Holzhey, Gero Strauss, Oliver Burgert, and Volkmar Falk. 2007. The Impact of Haptic Learning in Telemanipulatorassisted Surgery. Surgical Laparoscopy Endoscopy \& Percutaneous Techniques 17, 5 (Oct. 2007), 402. https://doi.org/10.1097/SLE. ob013e3180f60c23

[31] Seung-kook Jun, Pankaj Singhal, Madusudanan Sathianarayanan, Sudha Garimella, Abeer Eddib, and Venkat Krovi. 2012. Evaluation of Robotic Minimally Invasive Surgical Skills Using Motion Studies. In Proceedings of the Workshop on Performance Metrics for Intelligent Systems (PerMIS '12). ACM, New York, NY, USA, 198-205. https://doi.org/10.1145/2393091.2393129

[32] Louise H. Kidder and Michelle Fine. 1987. Qualitative and quantitative methods: When stories converge. New Directions for Program Evaluation 1987, 35 (1987), 57-75. https://doi.org/10.1002/ev.1459

[33] Fuji Lai and Eileen Entin. 2005. Robotic Surgery and the Operating Room Team. Proceedings of the Human Factors and Ergonomics Society Annual Meeting 49, 11 (Sept. 2005), 1070-1073. https://doi.org/10. $1177 / 154193120504901115$

[34] Thomas K Landauer. 1995. The Trouble With Computers: Usefulness, Usability and Productivity. The MIT Press Cambridge, Cambridge, MA.

[35] Anthony R. Lanfranco, Andres E. Castellanos, Jaydev P. Desai, and William C. Meyers. 2004. Robotic Surgery. Annals of Surgery 239, 1 (Jan. 2004), 14-21. https://doi.org/10.1097/01.sla.0000103020.19595.7d

[36] G. I. Lee, M. R. Lee, I. Green, M. Allaf, and M. R. Marohn. 2017. Surgeons' physical discomfort and symptoms during robotic surgery: a comprehensive ergonomic survey study. Surgical Endoscopy 31, 4 (2017), 1697-1706. https://doi.org/10.1007/s00464-016-5160-y

[37] L. Lingard, S. Espin, S. Whyte, G. Regehr, G. R. Baker, R. Reznick, J. Bohnen, B. Orser, D. Doran, and E. Grober. 2004. Communication failures in the operating room: an observational classification of recurrent types and effects. BMJ Quality \& Safety 13, 5 (Oct. 2004), 330-334. https://doi.org/10.1136/qshc.2003.008425

[38] Hongpeng Liu, Ying Xu, Lei Hu, Peifu Tang, and Lifeng Wang. 2018. An Investigation of Errors in Fluoroscopic Navigation for Robot-Assisted Orthopedic Surgery. In Proceedings of the 2018 8th International Conference on Bioscience, Biochemistry and Bioinformatics (ICBBB 2018). ACM New York, NY, USA, 134-138. https://doi.org/10.1145/3180382.3180394

[39] Wendy E. Mackay, Wendy E. Mackay, Visiting Professor, and Visiting Professor. 2000. Responding to cognitive overload: Co-adaptation between users and technology. Intellectica 30 (2000), 177-193.

[40] T. Manser. 2009. Teamwork and patient safety in dynamic domains of healthcare: a review of the literature. Acta Anaesthesiologica Scandinavica 53, 2 (Feb. 2009), 143-151. https://doi.org/10.1111/j.1399-6576. 2008.01717.x

[41] Jacques Marescaux, Joel Leroy, Francesco Rubino, Michelle Smith, Michel Vix, Michele Simone, and Didier Mutter. 2002. Transcontinental Robot-Assisted Remote Telesurgery: Feasibility and Potential Applications. Annals of Surgery 235, 4 (April 2002), 487-492. https://www.ncbi.nlm.nih.gov/pmc/articles/PMC1422462/

[42] S. Martin. 2004. The role of the first assistant in robotic assisted surgery. British journal of perioperative nursing : the journal of the National Association of Theatre Nurses 14, 4 (April 2004), 159-163. https://doi.org/10.1177/175045890401400403

[43] Helena M. Mentis, Amine Chellali, and Steven Schwaitzberg. 2014 Learning to See the Body: Supporting Instructional Practices in Laparoscopic Surgical Procedures. In Proceedings of the SIGCHI Conference on Human Factors in Computing Systems (CHI '14). ACM, New York, NY, USA, 2113-2122. https://doi.org/10.1145/2556288.2557387

[44] Helena M. Mentis, Kenton O’Hara, Gerardo Gonzalez, Abigail Sellen, Robert Corish, Antonio Criminisi, Rikin Trivedi, and Pierre Theodore.
2015. Voice or Gesture in the Operating Room. In Proceedings of the 33rd Annual ACM Conference Extended Abstracts on Human Factors in Computing Systems (CHI EA '15). ACM, New York, NY, USA, 773-780. https://doi.org/10.1145/2702613.2702963

[45] Helena M. Mentis, Ahmed Rahim, and Pierre Theodore. 2016. Crafting the Image in Surgical Telemedicine. In Proceedings of the 19th ACM Conference on Computer-Supported Cooperative Work \& Social Computing (CSCW' 16). ACM, New York, NY, USA, 744-755. https: //doi.org/10.1145/2818048.2819978

[46] Helena M. Mentis and Alex S. Taylor. 2013. Imaging the Body: Embodied Vision in Minimally Invasive Surgery. In Proceedings of the SIGCHI Conference on Human Factors in Computing Systems (CHI '13). ACM, New York, NY, USA, 1479-1488. https://doi.org/10.1145/2470654. 2466197

[47] K. Moorthy, Y. Munz, A. Dosis, J. Hernandez, S. Martin, F. Bello, T. Rockall, and A. Darzi. 2004. Dexterity enhancement with robotic surgery. Surgical Endoscopy And Other Interventional Techniques 18, 5 (May 2004), 790-795. https://doi.org/10.1007/s00464-003-8922-2

[48] Guillaume Morel, JÃlrãteme Szewczyk, and Marie-Aude Vitrani. 2013. Comanipulation. In Medical Robotics. Wiley-Blackwell, Hoboken, NJ, 303-350. https://doi.org/10.1002/9781118562147.ch9

[49] Janice M. Morse. 1997. âĂIJPerfectly Healthy, But DeadâĂİ: The Myth of Inter-Rater Reliability. Qualitative Health Research 7, 4 (1997), 445447. https://doi.org/10.1177/104973239700700401

[50] B. R. Nair, J. L. Coughlan, and M. J. Hensley. 1998. Impediments to bed-side teaching. Medical education 32, 2 (March 1998), 159-162. https://doi.org/10.1046/j.1365-2923.1998.00185.x

[51] R. Nayyar, S. Yadav, P. Singh, and P. N. Dogra. 2016. Impact of assistant surgeon on outcomes in robotic surgery., Impact of assistant surgeon on outcomes in robotic surgery. Indian journal of urology : IfU : journal of the Urological Society of India, Indian fournal of Urology: IfU: Fournal of the Urological Society of India 32, 32, 3, 3 (Sept. 2016), 204, 204-209. https://doi.org/10.4103/0970-1591.185095,10.4103/0970-1591.185095

[52] E. Neugebauer, H. Troidl, C. K. Kum, E. Eypasch, M. Miserez, and A. Paul. 1995. The E.A.E.S. Consensus development conferences on laparoscopic cholecystectomy, appendectomy, and hernia repair. Surgical Endoscopy 9, 5 (May 1995), 550-563. https://doi.org/10.1007/BF00206852

[53] Ilana Nisky, Felix Huang, Amit Milstein, Carla M. Pugh, Ferdinando A. Mussa-ivaldi, and Amir Karniel. 2012. Perception of Stiffness in Laparoscopy âĂŞ the Fulcrum Effect. Studies in health technology and informatics 173 (2012), 313-319. https://www.ncbi.nlm.nih.gov/pmc/ articles/PMC4102265/

[54] Anne-Sophie Nyssen and AdÃllaÃŕde Blavier. 2009. Verbal Communication As a Sign of Adaptation in Socio-technical Systems: The Case of Robotic Surgery. In Proceedings of the 9th Bi-annual International Conference on Naturalistic Decision Making (NDM'09). BCS Learning \& Development Ltd., Swindon, UK, 39-39. http://dl.acm.org/citation. $\mathrm{cfm}$ ?id $=2228107.2228149$

[55] J. H. Obradovich and D. D. Woods. 1996. Users as designers: how people cope with poor HCI design in computer-based medical devices. Human Factors 38, 4 (Dec. 1996), 574-592. https://doi.org/10.1518/ 001872096778827251

[56] Adrian Park, Gyusung Lee, F. Jacob Seagull, Nora Meenaghan, and David Dexter. 2010. Patients Benefit While Surgeons Suffer: An Impending Epidemic. fournal of the American College of Surgeons 210, 3 (March 2010), 306-313. https://doi.org/10.1016/j.jamcollsurg.2009.10. 017

[57] Resad P. Pasic, John A. Rizzo, Hai Fang, Susan Ross, Matt Moore, and Candace Gunnarsson. 2010. Comparing Robot-Assisted with Conventional Laparoscopic Hysterectomy: Impact on Cost and Clinical Outcomes. Fournal of Minimally Invasive Gynecology 17, 6 (Nov. 2010), 730-738. https://doi.org/10.1016/j.jmig.2010.06.009 
[58] D. a. G. Reyes, B. Tang, and A. Cuschieri. 2006. Minimal access surgery (MAS)-related surgeon morbidity syndromes. Surgical Endoscopy And Other Interventional Techniques 20, 1 (Jan. 2006), 1. https://doi.org/10. 1007/s00464-005-0315-2

[59] Craig G. Rogers, Rajesh Laungani, Akshay Bhandari, Louis Spencer Krane, Daniel Eun, Manish N. Patel, Ronald Boris, Alok Shrivastava, and Mani Menon. 2009. Maximizing Console Surgeon Independence during Robot-Assisted Renal Surgery by Using the Fourth Arm and TileProâĎć. Fournal of Endourology 23, 1 (Jan. 2009), 115-122. https: //doi.org/10.1089/end.2008.0416

[60] S. Saunders. 2004. Why good communication skills are important for theatre nurses. Nursing times 100, 14 (2004), 42-44. http://europepmc. org/abstract $/ \mathrm{med} / 15119134$

[61] Lauren Schiff, Ziv Tsafrir, Joelle Aoun, Andrew Taylor, Evan Theoharis, and David Eisenstein. 2016. Quality of Communication in Robotic Surgery and Surgical Outcomes. FSLS : Journal of the Society of Laparoendoscopic Surgeons 20, 3 (2016), e2016.00026. https: //doi.org/10.4293/JSLS.2016.00026

[62] H. W. R. Schreuder, R. Wolswijk, R. P. Zweemer, M. P. Schijven, and R. H. M. Verheijen. 2012. Training and learning robotic surgery, time for a more structured approach: a systematic review. BfOG: An International fournal of Obstetrics \& Gynaecology 119, 2 (Jan. 2012), 137-149. https://doi.org/10.1111/j.1471-0528.2011.03139.x

[63] Anna Serur, Rebecca Rhee, and Matthew M. Philp. 2015. The Role of a Bedside Assistant. In Robotic Approaches to Colorectal Surgery, Howard Ross, Sang Lee, Bradley J. Champagne, Alessio Pigazzi, and David E. Rivadeneira (Eds.). Springer International Publishing, Cham, 111-117. https://doi.org/10.1007/978-3-319-09120-4_9

[64] J. B. Sexton, M. A. Makary, A. R. Tersigni, D. Pryor, A. Hendrich, E. J. Thomas, C. G. Holzmueller, A. P. Knight, Y. Wu, and P. J. Pronovost. 2006. Teamwork in the operating room: frontline perspectives among hospitals and operating room personnel. Anesthesiology 105, 5 (Nov. 2006), 877-884. https://doi.org/10.1097/00000542-200611000-00006
[65] Olivia Sgarbura and Catalin Vasilescu. 2010. The decisive role of the patient-side surgeon in robotic surgery. Surgical Endoscopy 24, 12 (Dec. 2010), 3149-3155. https://doi.org/10.1007/s00464-010-1108-9

[66] Karem Slim and Michel Canis. 2017. La chirurgie robotique. Peut-on (doit-on) nager Ãă contre-courant ? La Presse MÃldicale 46, 6 (June 2017), 557 - 560. https://doi.org/10.1016/j.lpm.2017.04.004

[67] Chandru P. Sundaram, Michael O. Koch, Thomas Gardner, and Jonathan E. Bernie. 2005. Utility of the fourth arm to facilitate robotassisted laparoscopic radical prostatectomy. BfU international 95, 1 (Jan. 2005), 183-186. https://doi.org/10.1111/j.1464-410X.2005.05274.x

[68] Intuitive Surgical. 2016. Intuitive Surgical Investor Presentation Q2 2016. https://isrg.intuitive.com/news-releases/news-release-details/ intuitive-surgical-announces-preliminary-fourth-quarter-and-1

[69] Frank Tendick, Russell W. Jennings, Gregory Tharp, and Lawrence Stark. 1993. Sensing and Manipulation Problems in Endoscopic Surgery: Experiment, Analysis, and Observation. Presence: Teleoperators and Virtual Environments 2, 1 (Jan. 1993), 66-81. https: //doi.org/10.1162/pres.1993.2.1.66

[70] Charlotte Tsui, Rachel Klein, and Matthew Garabrant. 2013. Minimally invasive surgery: national trends in adoption and future directions for hospital strategy. Surgical Endoscopy 27, 7 (July 2013), 2253-2257. https://doi.org/10.1007/s00464-013-2973-9

[71] Shabnam Undre, Andrew N. Healey, Ara Darzi, and Charles A. Vincent. 2006. Observational Assessment of Surgical Teamwork: A Feasibility Study. World fournal of Surgery 30, 10 (Oct. 2006), 1774-1783. https: //doi.org/10.1007/s00268-005-0488-9

[72] Jessica L. Webster and Caroline G. L. Cao. 2006. Lowering communication barriers in operating room technology. Human Factors 48, 4 (2006), 747-758. https://doi.org/10.1518/001872006779166271

[73] Terry Winograd, Fernando Flores, and Fernando F Flores. 1986. Understanding computers and cognition: A new foundation for design. Intellect Books, Ablex, Norwood, NJ.

[74] Bertram Yuh. 2013. The bedside assistant in robotic surgery-keys to success. Urologic Nursing 33, 1 (Feb. 2013), 29-32. 